Fatima Zohra Elamrani, Khalil Ezzinbi*, and Samir Fatajou

\title{
Eberlein weak almost periodic solutions for a class of integro-differential equations with infinite delay
}

https://doi.org/10.1515/msds-2018-0010

Received April 14, 2017; accepted October 4, 2018

\begin{abstract}
In this work, we provide sufficient conditions ensuring the existence and uniqueness of an Eberlein weakly almost periodic solutions for some semilinear integro-differential equations with infinite delay in Banach spaces. For illustration, we provide an example arising in viscoelasticity theory.
\end{abstract}

Keywords: Integro-differential equation, Eberlein weak almost periodic solution, immediately norm continuous $C_{0}$-semigroup

\section{Introduction}

In this work, we study the existence and uniqueness of an Eberlein weakly almost periodic solution for the following integro-differential equation with infinite delay

$$
\frac{d}{d t} x(t)=A x(t)+\alpha \int_{-\infty}^{t} e^{-\beta(t-s)} A x(s) d s+f(t, x(t)), \quad t \in \mathbb{R},
$$

where $A: D(A) \rightarrow X$ is the generator of an immediately norm continuous $C_{0}$-semigroup defined on a Banach space $X$ and $f: \mathbb{R} \times X \rightarrow X$ is continuous. Under appropriate assumptions on the scalars $\alpha, \beta$, the operator $A$ and the forcing function $f$ we prove that the equation (1) has a unique bounded mild solution $x($.) which behaves in the same way that $f$ does. Precisely, we give conditions implying that $x($.) is Eberlein weak almost periodic if $f(., x)$ is Eberlein weak almost periodic for all $x \in X$.

Integro-differential equation becomes now an interesting field in dynamical systems and have many applications in physical systems, like viscoelasticity of heat conduction with memory, and in such applications the operator $A$ typically is the Laplacian in $X=L^{2}(\Omega)$. The exponential kernel $\alpha e^{-\beta t}$ is the typical choice when one consider Maxwell materials in viscoelasticity theory. More details about this integro-differential equation and its several applied fields can be fond in $[9,23,29]$ and the references therein.

The problem, of existence and uniqueness of periodic, almost periodic, almost automorphic and pseudo almost periodic solutions for the type of nonlinear evolution equations (1) has been extensively studied in the literature; see [10, 12, 13, 20-22, 24-28] and references therein.

The organization of this work is as follows: in Section 2, we recall some preliminaries results on Eberlin weakly almost periodic functions. In section 2, firstly, we prove the existence of an Eberlin weakly almost solution for nonhomogeneous linear equations, secondly, we prove the existence and uniqueness an Eberlin

Fatima Zohra Elamrani: Départment de Mathématiques et Informatique, E. S. T. Safi Université Cadi Ayyad, Route Dar Si Aissa BP 89 Safi, Morocco, E-mail: fzelamrani96@gmail.com

*Corresponding Author: Khalil Ezzinbi: Départment de Mathématiques, Faculté des Sciences Semlalia, Université Cadi Ayyad , P.O. Box 2390, Marrakesh, Morocco - UMI 209 UMMISCO. IRD UPMC., E-mail: ezzinbi@gmail.com Samir Fatajou: Laboratoire L. M. C.; Départment de Mathématiques et Informatique, Faculté Polydisciplinaire-Safi (FPS), Université Cadi Ayyad, Sidi Bouzid B. P. 4162, Safi, Morocco - UMI 209 UMMISCO. IRD UPMC., E-mail: fatajou@yahoo.fr 
weakly almost solution of nonlinear equation by using the strict contraction principle. Last Section is devoted to some illustration, we propose to study the existence and uniqueness of an Eberlin weakly almost solution for some model arising in viscoelasticity theory

\section{Eberlein weakly almost periodic functions}

Let $(X,\|\cdot\|)$ be a Banach space, $\mathbb{B}(X), C(\mathbb{R}, X)$ and $B C(\mathbb{R}, X)$ denote respectively the space of bounded linear operators from $X$ to $X$, the space of continuous functions from $\mathbb{R}$ into $X$, and the space of bounded continuous functions from $\mathbb{R}$ into $X$, respectively. Note that $\left(B C(\mathbb{R}, X),\|\cdot\|_{\infty}\right)$ provided with the sup norm

$$
\|f\|_{\infty}:=\sup _{t \in \mathbb{R}}\|f(t)\|_{X}
$$

for each $f \in B C(\mathbb{R}, X)$, is a Banach space.

The concept of almost periodic function was introduced by Bohr [6] in 1925 by a criterion of Bochner [5], we say that a function $f \in B C(\mathbb{R}, X)$ is almost periodic, if its orbit given by:

$$
O_{\mathbb{R}}(f):=\left\{f_{\tau}:=\{t \rightarrow f(t+\tau)\}: \tau \in \mathbb{R}\right\}
$$

is relatively compact with respect to the uniform topology of the sup-normed Banach space $B C(\mathbb{R}, X)$. The canonical weakening of the above definition leads to the notion of Eberlein-weakly almost periodic functions, as done by Eberlein [8] in 1949 : a function $f \in B C(\mathbb{R}, X)$, is said to be Eberlein-weakly almost periodic, if the orbit of $f$ is relatively compact with respect to the weak topology of the sup-normed Banach space $B C(\mathbb{R}, X)$. For the sequel, $A P(\mathbb{R}, X), W(\mathbb{R}, X)$ and $W R C(\mathbb{R}, X)$ will denote the space of all almost periodic $X$-valued functions, the space of all Eberlein-weakly almost periodic $X$-valued functions and the space of all Eberlein-weakly almost periodic $X$-valued functions with a relatively compact range respectively. The concept of Eberlein-weak almost periodicity, is then larger than almost periodicity in the sense of Bochner. Obviously we have

$$
A P(\mathbb{R}, X) \subset W(\mathbb{R}, X) .
$$

The concept of Eberlein-weak almost periodicity is especially useful in harmonic analysis, (cf. [8, 17, 30]), in the theory of topological semigroups, where we only cite the book of Berglund, Junghenn and Milnes [4], and, more recently, in differential equations [1-3, 31-33].

In $[14,15]$ Deleeuw and Glicksberg proved that, if we consider the subspace of Eberlein-weakly almost periodic functions, which contains zero in the weak closure of the orbit (weak topology of $\left(B C(\mathbb{R}, X),\|\cdot\|_{\infty}\right)$, namely:

$$
W_{0}(\mathbb{R}, X):=\left\{f \in W(\mathbb{R}, X): \text { for a sequence }\left(s_{n}\right)_{n \in \mathbb{N}} \subseteq \mathbb{R}, f_{s_{n}} \rightarrow 0\right\} .
$$

Then every Eberlein-weakly almost periodic function $f$ is represented uniquely by the form $f=f^{a}+f_{0}$, where $f^{a}$ (the principal term) is an almost periodic function and $f_{0}$ (the $W_{0}$-perturbation) is in $W_{0}(\mathbb{R}, X)$. Moreover,

$$
\lim _{T \rightarrow+\infty} \frac{1}{T} \int_{0}^{T}\left(f_{t}(s)-f_{t}^{a}(s)\right) d s=0,
$$

uniformly in $t \in \mathbb{R}$.

For a more details on the decomposition and the ergodic results we refer to [19], and [34].

In order to prove the weak compactness of the translates, Ruess and Summers extended the double limits criterion of Grothendieck [18] to the following form:

Proposition 2.1. A subset $H \subset B C(\mathbb{R}, X)$, is relatively weakly compact if and only if the following are true: 1. $H$ is bounded in $B C(\mathbb{R}, X)$, 
2. for all $\left(h_{m}\right)_{m \in \mathbb{N}} \subseteq H,\left(t_{n}\right)_{n \in \mathbb{N}} \subseteq \mathbb{R}$, and $\left(x_{n}^{\star}\right)_{n \in \mathbb{N}} \subseteq B_{X^{\star}}$ the following double limits condition holds :

$$
\lim _{m \rightarrow \infty} \lim _{n \rightarrow \infty}\left\langle h_{m}\left(t_{n}\right), x_{n}^{\star}\right\rangle=\lim _{n \rightarrow \infty} \lim _{m \rightarrow \infty}\left\langle h_{m}\left(t_{n}\right), x_{n}^{\star}\right\rangle,
$$

whenever the iterated limits exist.

The above result is the main working tools to prove the weak almost periodicity.

In order to give sufficient conditions for the existence of Eberlein weakly almost periodic solutions to semilinear integro-differential equations, we need the following definition, where $(D, \tau)$ is assumed to be a topological space.

Definition 2.2. A continuous function $f: \mathbb{R} \times D \longrightarrow X$ is said to be Eberlein weakly almost periodic in $t$ uniformly with respect to $x$ in $D$ if the following are true:

1. $\forall x \in X, f(., x) \in W(\mathbb{R}, X)$; and

2. for each compact subset $K \subset D$ such that $\left(K, \tau_{K}\right)$ is metric,

$$
\begin{aligned}
\rho_{K} \quad: \quad K & \rightarrow W(\mathbb{R}, X) \\
x & \mapsto f(., x)
\end{aligned}
$$

is continuous.

For the sequel, $W(\mathbb{R} \times D, X)$ denotes the set of such functions.

Theorem 2.3. Suppose that $D=\left(X, \sigma\left(X, X^{\prime}\right)\right)$, where $\sigma\left(X, X^{\prime}\right)$ denotes the weak topology, $x(.) \in W(\mathbb{R}, X)$ and $f \in W(\mathbb{R} \times D, X)$, then $\{t \mapsto f(t, x(t))\} \in W(\mathbb{R}, X)$.

Proof. In order to prove that

$$
\{t \longmapsto f(t, x(t))\}
$$

is Eberlein weakly almost periodic, by proposition (2.1), we have to verify that for given sequences $\left(w_{n}\right)_{n \in \mathbb{N}} \subset$ $\mathbb{R},\left(t_{m}\right)_{m \in \mathbb{N}} \subset \mathbb{R}$, and $\left(x_{m}^{*}\right)_{m \in \mathbb{N}} \subset B_{X^{*}}$,

$$
\lim _{n \rightarrow \infty} \lim _{m \rightarrow \infty}\left\langle f\left(t_{m}+w_{n}, x\left(t_{m}+w_{n}\right)\right), x_{m}^{\star}\right\rangle=\lim _{m \rightarrow \infty} \lim _{n \rightarrow \infty}\left\langle f\left(t_{m}+w_{n}, x\left(t_{m}+w_{n}\right)\right), x_{m}^{\star}\right\rangle,
$$

whenever the iterated limits exist.

Assuming that the iterated limits exist and by the fact that we only have to prove the equality of them, we may pass to subsequences for the verification.

As from [[16], V. 6.3., pp.434], we recall, that the weak topology on weakly compact subsets in separable Banach spaces is a metric topology. Noting that continuous images of separable spaces are separable, we obtain $\overline{\operatorname{span}}\{x(\mathbb{R})\}$, is separable, hence the weak topology on $K:=\overline{x(\mathbb{R})}^{\sigma}$ is metric, where $\sigma$ denotes the weak topology. Thus without loss of generality, we may assume that $\left(x\left(t_{m}+w_{n}\right)\right)_{n, m \in \mathbb{N}}$ satisfies the double limits conditions. Let $x_{0} \in K$ be the double limit. We define:

$$
\begin{aligned}
b_{n, m} & :=\left\langle f\left(t_{m}+w_{n}, x\left(t_{m}+w_{n}\right)\right), x_{m}^{\star}\right\rangle, \\
b & :=\lim _{n \rightarrow \infty} \lim _{m \rightarrow \infty}\left\langle f\left(t_{m}+w_{n}, x\left(t_{m}+w_{n}\right)\right), x_{m}^{\star}\right\rangle, \text { and } \\
a_{n, m} & :=\left\langle f\left(t_{m}+w_{n}, x_{0}\right), x_{m}^{\star}\right\rangle,
\end{aligned}
$$

Since $t \longmapsto f\left(t, x_{0}\right)$ is Eberlein weakly almost periodic, without loss of generality we can assume that $\left(a_{n, m}\right)_{n, m \in \mathbb{N}}$ satisfies the double limits condition. Let $a \in \mathbb{R}$ be the double limits.

We have

$$
|b-a| \leq\left|b-b_{n, m}\right|+\left|b_{n, m}-a_{n, m}\right|+\left|a_{n, m}-a\right| .
$$


From the convergence of $\left(a_{n, m}\right)_{n, m \in \mathbb{N}}$ and $\left(b_{n, m}\right)_{n, m \in \mathbb{N}}$, we derive that for every $\varepsilon>0$, there exists $n_{0} \in \mathbb{N}$, such that for all $n \geq n_{0}$, there is an $m_{n}^{0}$, such that

$$
\begin{aligned}
& \left|a_{n, m}-a\right|<\frac{\varepsilon}{3} \quad \forall m \geq m_{n}^{0}, \text { and } \\
& \left|b_{n, m}-b\right|<\frac{\varepsilon}{3} \quad \forall m \geq m_{n}^{0} .
\end{aligned}
$$

Let $d$ denote the metric on $K:=\overline{\chi(\mathbb{R})}^{\sigma}$. Using the double limits condition of the sequence $\left(x\left(t_{m}+w_{n}\right)\right)_{n, m \in \mathbb{N}}$, for a given $\eta$ there exists $n_{1} \in \mathbb{N}$, such that for all $n \geq n_{1}$, there is an $m_{n}^{1}$, such that $d\left(x\left(t_{m}+w_{n}\right), x_{0}\right)<$ $\eta$, for all $m \geq m_{n}^{1}$. From the continuity of the map

$$
\begin{aligned}
\rho_{K}: K & \rightarrow W(\mathbb{R}, X) \\
& \quad \mapsto f(., x)
\end{aligned},
$$

for $\varepsilon>0$, we find a $\eta>0$, and according to the previous remark, there is an $n_{1} \in \mathbb{N}$, such that for all $n \geq n_{1}$, we found an $m_{n}^{1}$, with

$$
\left\|f\left(., x\left(t_{m}+w_{n}\right)\right)-f\left(., x_{0}\right)\right\|<\frac{\varepsilon}{3}, \text { for all } m \geq m_{n}^{1} .
$$

This yields $|b-a|<\varepsilon$, and hence $b=a$. Noting that the same routine works for the interchanged limit of $\left(b_{n, m}\right)_{n, m \in \mathbb{N}}$, the theorem is proved.

For the sequel, $W R C(\mathbb{R} \times D, X):=\{f \in W(\mathbb{R} \times D, X): f(., x) \in W R C(\mathbb{R}, X), \forall x \in D\}$.

Corollary 2.4. Let, for a Banach space $Y, D=\left(Y,\|\cdot\|_{Y}\right)$, and $f \in W(\mathbb{R} \times D, X)$. Then, for any given $x(.) \in$ $W(\mathbb{R}, Y)$ with a relatively compact range, $\{t \mapsto f(t, x(t))\} \in W(\mathbb{R}, X)$. Moreover, if $f \in W R C(\mathbb{R} \times D, X)$, $t \mapsto f(t, x(t))$ is Eberlein weakly almost periodic and its range is relatively compact.

Proof. We apply the previous theorem to $K:=\overline{\chi(\mathbb{R})}\|\cdot\|$, since $(K, \sigma)=(K,\|\cdot\|)$. For the second part, it remains to prove the compactness of

$$
\{f(t, x(t)): t \in \mathbb{R}\} .
$$

Thus for a given sequence $\left(t_{n}\right)_{n \in \mathbb{N}}$, we have to find a subsequence $\left(t_{n_{k}}\right)_{k \in \mathbb{N}}$ such that

$$
\left(f\left(t_{n_{k}}, x\left(t_{n_{k}}\right)\right)\right)_{k \in \mathbb{N}}
$$

is convergent in $X$. Since $x($.$) has compact range, without loss of generality we can assume that$

$$
x\left(t_{n}\right) \rightarrow x
$$

For this $x \in Y$, we have choose a subsequence such that

$$
f\left(t_{n_{k}}, x\left(t_{n_{k}}\right)\right) \rightarrow y,
$$

for some $y \in Y$. From the continuity of $\rho_{\{x(t): t \in \mathbb{R}\}}$, we obtain

$$
f\left(., x\left(t_{n_{k}}\right)\right) \rightarrow f(., x)
$$

uniformly on $\mathbb{R}$. Thus

$$
\begin{aligned}
\left\|f\left(t_{n_{k}}, x\left(t_{n_{k}}\right)\right)-y\right\| & \left.\left.\leq \| f\left(t_{n_{k}}, x\left(t_{n_{k}}\right)\right)-f\left(t_{n_{k}}, x\right)\right)\|+\| f\left(t_{n_{k}}, x\right)\right)-y \| \\
& \left.\left.\leq \| f\left(., x\left(t_{n_{k}}\right)\right)-f(., x)\right)\left\|_{\infty}+\right\| f\left(t_{n_{k}}, x\right)\right)-y \|,
\end{aligned}
$$

and the proof is complete. 


\section{Eberlein weakly almost periodic solutions integro-differential equations in Banach spaces}

Now, to equation (1), we associate the following Cauchy problem:

$$
\left\{\begin{array}{l}
\frac{d}{d t} x(t)=A x(t)+\alpha \int_{-\infty}^{t} e^{-\beta(t-s)} A x(s) d s, t \geq 0, \\
x(0)=x_{0},
\end{array}\right.
$$

where $A$ is the infinitesimal generator of $C_{0}$-semigroup on $X$.

In the sequel, We say that a solution of (2) is uniformly exponentially bounded if for some $\in \mathbb{R}$, there exist constants $\omega>0, M \geq 1$ such that for each $x_{0} \in D(A)$, the corresponding solution $x$ satisfies:

$$
\|x(t)\| \leq M e^{-\omega t}\left\|x_{0}\right\| \text { for } t \geq 0 .
$$

In particular, we say that the solutions of (2) are uniformly exponentially stable if (3) holds for some $\omega>0, M \geq 1$.

Definition 3.1. A function $S: \mathbb{R}^{+} \rightarrow \mathbb{B}(X)$ strongly continuous is said to be immediately norm continuous if $S:(0, \infty) \rightarrow \mathbb{B}(X)$ is continuous.

The following results are taken from ([7]).

Theorem 3.2. Let $\alpha \neq 0, \beta>0$ with $\alpha+\beta>0$ be given. Assume that

$\left(H_{1}\right)$ : A generates an immediately norm continuous $C_{0}$-semigroup on $X$;

$\left(H_{2}\right): \sup \left\{\operatorname{Re}(\lambda), \lambda \in \mathbb{C}: \lambda(\lambda+\beta)(\lambda+\alpha+\beta)^{-1} \in \sigma(A)\right\}<0$.

Then, the following are true

1. For each $x \in D(A)$, the equation (2) has a unique solution $x($.$) .$

2. The solutions of the equation (2) are uniformly exponentially stable.

In the sequel, we study the existence and uniqueness of an Eberlein weak almost periodic solution for the following nonhomogeneous equation:

$$
\frac{d}{d t} x(t)=A x(t)+\alpha \int_{-\infty}^{t} e^{-\beta(t-s)} A x(s) d s+f(t) \text { for } t \in \mathbb{R},
$$

where $\alpha, \beta \in \mathbb{R}$ and $A$ generates an immediately norm continuous $C_{0}$-semigroup on a Banach space $X$ and $f: \mathbb{R} \rightarrow X$ is a continuous function satisfying some additional conditions that will be defined in the next. As a consequence of theorem (3.2), we have the following result where we deduce that it is possible to construct for (4) a strongly continuous family of bounded and linear operators, that commutes with $A$ and satisfy the resolvent equation, more details, we refer to [29].

Theorem 3.3. Let $\alpha \neq 0, \beta>0$ with $\alpha+\beta>0$. Assume that $\left(H_{1}\right)$ and $\left(H_{2}\right)$ hold. Then, there exists a uniformly exponentially stable and strongly continuous family of operators $(U(t))_{t \geq 0} \subset \mathbb{B}(X)$ such that

1. $U(t) D(A) \subset D(A), \forall t \geq 0$.

2. $U(t) A x=A U(t) x, \forall t \geq 0, x \in D(A)$. 
3. $U(t) x=\int_{0}^{t} \kappa(t-s) A U(s) x d s, \forall t \geq 0$ and $x \in X$,

where $\kappa(t):=1+\frac{\alpha}{\beta}\left(1-e^{-\beta t}\right), t \geq 0$.

Proof. Let $t \geq 0$. We define the operator $U(t)$ by:

$$
U(t) x=x(.),
$$

where $x($.$) , is the unique solution of (2) such that x(0)=x$. We have that $t \mapsto U(t) x$ is differentiable and satisfies

$$
\frac{d}{d t} U(t) x=A U(t) x+\alpha \int_{0}^{t} e^{-\beta(t-s)} A U(s) x d s .
$$

From Fubini's theorem we found that :

$$
\begin{aligned}
U(t) x-x & =\int_{0}^{t} A U(s) x d s+\alpha \int_{0}^{t} \int_{0}^{s} e^{-\beta(s-r)} A U(r) x d r d s \\
& =\int_{0}^{t} A U(s) x d s+\alpha \int_{0}^{t} \int_{r}^{t} e^{-\beta(s-r)} A U(r) x d s d r \\
& =\int_{0}^{t} A U(s) x d s+\alpha \int_{0}^{t} \int_{0}^{t} e^{-\beta \tau} A U(r) x d \tau d r \\
& =\int_{0}^{t} A U(s) x d s+\frac{\alpha}{\beta} \int_{0}^{t}\left(1-e^{-\beta(t-r)}\right) A U(r) x d r \\
& =\int_{0}^{t} 1+\frac{\alpha}{\beta}\left(1-e^{-\beta(t-r)}\right) A U(r) x d r \\
& =\int_{0}^{t} \kappa(t-r) A U(r) x d r
\end{aligned}
$$

Lemma 3.4. Under assumptions $\left(H_{1}\right)$ and $\left(H_{2}\right)$, if $f: \mathbb{R} \rightarrow X$ is a bounded continuous function, then equation (4) has a unique bounded mild solution $x($.) on $\mathbb{R}$ which is given by

$$
x(t)=\int_{-\infty}^{t} U(t-r) f(r) d r, \forall t \in \mathbb{R},
$$

where $(U(t))_{t \geq 0}$ is given in Theorem (3.3).

Proof. Since $U$ satisfies the resolvent equation

$$
U(t) x=\int_{0}^{t} \kappa(t-s) A U(s) x d s, \forall x \in X,
$$

where $\kappa(t):=1+\frac{\alpha}{\beta}\left(1-e^{-\beta t}\right), t \geq 0$. We have that $\kappa($.$) is differentiable and the above equation shows that for$ each $x \in X, \frac{d}{d t} U(t) x$ exists and

$$
\frac{d}{d t} U(t) x=A U(t) x+\alpha \int_{0}^{t} e^{-\beta(t-s)} A U(s) x d s .
$$


Its remains to prove that $u$ is a mild solution of equation (4). Since $A$ is a closed operator, using Fubini's theorem, we have

$$
\begin{aligned}
\frac{d}{d t} u(t) & =U(0) f(t)+\int_{-\infty}^{t} \frac{d}{d t} U(t-s) f(s) d s \\
& =f(t)+\left[\int_{-\infty}^{t} A U(t-s) f(s)+\alpha \int_{0}^{t} e^{-\beta(t-s-r)} A U(r) f(s) d r\right] d s \\
& =f(t)+\int_{-\infty}^{t} A U(t-s) f(s) d s+\alpha \int_{-\infty}^{t-s} \int_{0}^{t} e^{-\beta(t-s-r)} A U(r) f(s) d r d s \\
& =f(t)+A u(t)+\alpha \int_{-\infty}^{t} \int_{s}^{t} e^{-\beta(t-r)} A U(r-s) f(s) d r d s \\
& =f(t)+A u(t)+\alpha \int_{-\infty}^{t} e^{-\beta(t-r)} \int_{-\infty}^{r} A U(r-s) f(s) d s d r \\
& =f(t)+A u(t)+\alpha \int_{-\infty} e^{-\beta(t-r)} A u(r) d r
\end{aligned}
$$

Note that, if $u(t) \in D(A), \forall t \in \mathbb{R}$, then a mild solution is a strong solution.

Theorem 3.5. Under assumptions $\left(H_{1}\right)$ and $\left(H_{2}\right)$, if $f$ is Eberlein weakly almost periodic with a relatively compact range. Then equation (4) has a unique bounded solution in $W R C(\mathbb{R}, X)$.

For the proof, the following lemma is needed.

Lemma 3.6. Let $\left(f_{n}\right)_{n \in \mathbb{N}} \subset B C(\mathbb{R}, X)$. Assume that there exists a compact set $K$ in $X$ such that

$$
\left\{f_{n}(t): n \in \mathbb{N} \text { and } t \in \mathbb{R}\right\} \subset K,
$$

and $f_{n} \rightarrow 0$ in $B C(\mathbb{R}, X)$. Then $\left\|f_{n}().\right\| \rightarrow 0$ in $B C(\mathbb{R})$.

Proof. Firstly, we show that the set $\left\{\left\|f_{n}().\right\|: n \in \mathbb{N}\right\}$ is weakly relatively compact in $B C(\mathbb{R})$.

Thus, from proposition (2.1), for given sequences $\left(n_{j}, t_{m}\right)_{j, m \in \mathbb{N}} \subset \mathbb{N} \times \mathbb{R}$, we have to verify the following identity :

$$
\lim _{j \rightarrow \infty} \lim _{m \rightarrow \infty}\left\|f_{n_{j}}\left(t_{m}\right)\right\|=\lim _{m \rightarrow \infty} \lim _{j \rightarrow \infty}\left\|f_{n_{j}}\left(t_{m}\right)\right\|
$$

whenever the iterated limits exist.

Since $\left\{f_{n_{j}}\left(t_{m}\right): j, m \in \mathbb{N}\right\} \subset K$, a diagonalization argument gives us subsequences, such that the iterated limits exist for the sequence $\left(f_{n_{j_{k}}}\left(t_{m_{l}}\right)\right)_{k, l \in \mathbb{N}}$. By the fact that we only have to verify the equality of the iterated limits, we may pass to these sequences. In order to avoid subindices, we may assume that the iterated limits exist for $\left(f_{n_{j}}\left(t_{m}\right)\right)_{j, m \in \mathbb{N}}$. The characterization of weak compactness gives that

$$
\lim _{j \rightarrow \infty} \lim _{m \rightarrow \infty} f_{n_{j}}\left(t_{m}\right)=\lim _{m \rightarrow \infty} \lim _{j \rightarrow \infty} f_{n_{j}}\left(t_{m}\right) .
$$

Since the convergence holds in norm, we proved that $\left\{\left\|f_{n}().\right\|: n \in \mathbb{N}\right\}$ is weakly relatively compact in $B C(\mathbb{R})$. Using that $\lim _{n \rightarrow \infty}\left\|f_{n}().\right\|=0$ a standard trick of topology gives $\left\|f_{n}().\right\| \rightarrow 0$ in $B C(\mathbb{R})$.

Proof. (of theorem) According to lemma (3.4), the only bounded mild solution of equation (4) is given by

$$
x(t)=\int_{-\infty}^{t} U(t-r) f(r) d r, \forall t \in \mathbb{R} .
$$


To prove that $x\left(\right.$.) is Eberlein weakly almost periodic, we use proposition (2.1). Therefore, let $\left(w_{n}\right)_{n \in \mathbb{N}} \subset \mathbb{R}$ and $\left(t_{m}, x_{m}^{\star}\right)_{m \in \mathbb{N}} \subset \mathbb{R} \times B_{X^{\star}}$ be given, such that the following iterated limits exist

$$
\begin{aligned}
\alpha & =\lim _{m \rightarrow \infty} \lim _{n \rightarrow \infty}\left\langle x\left(w_{n}+t_{m}\right), x_{m}^{\star}\right\rangle \\
\beta & =\lim _{n \rightarrow \infty} \lim _{m \rightarrow \infty}\left\langle x\left(w_{n}+t_{m}\right), x_{m}^{\star}\right\rangle .
\end{aligned}
$$

We have to show that $\alpha=\beta$.

Noting that only equality of the double limits has to be shown, we pass to subsequences.

Since every Eberlein weakly almost periodic function is uniformly continuous, hence $t \mapsto f_{t}$ is strongly continuous on $\mathbb{R}$. Nothing that continuous images of separable spaces are separable, we obtain $Y_{f}:=\overline{\operatorname{span}}\{f(\mathbb{R})\}$ is separable. As from [16], we recall, that the weak topology on a weakly compact subset in a separable Banach space is a metric space. We deduce that the weak topology on ${\overline{O_{\mathbb{R}}(f)}}^{\sigma}$ is metric, where $\sigma$ denotes the weak topology. As a consequence, we may pass to subsequence $\left(w_{n_{k}}\right)_{k \in \mathbb{N}}$ of $\left(w_{n}\right)_{n \in \mathbb{N}}$, such that the limit of $\left(f_{w_{n_{k}}}(.)\right)_{k \in \mathbb{N}}$ exist in the weak topology of $B C(\mathbb{R}, X)$, and without loss of generality, we may assume that

$$
f_{w_{n}} \rightarrow g
$$

where $g \in W(\mathbb{R}, X)$ has a relatively compact range.

Then, wee have that

$$
\begin{aligned}
\left\|x\left(t_{m}+w_{n}\right)-x\left(t_{m}+w_{k}\right)\right\| & \leq \int_{-\infty}^{t_{m}}\left\|U\left(t_{m}-\tau\right)\left(f\left(\tau+w_{n}\right)-f\left(\tau+w_{k}\right)\right)\right\| d \tau \\
& \leq M \int_{-\infty}^{t_{m}} e^{-\omega\left(t_{m}-\tau\right)}\left\|f\left(\tau+w_{n}\right)-g(\tau)\right\| d \tau+M \int_{-\infty}^{t_{m}} e^{-\omega\left(t_{m}-\tau\right)}\left\|f\left(\tau+w_{k}\right)-g(\tau)\right\| d \tau .
\end{aligned}
$$

Thus,

$$
\left\|x\left(t_{m}+w_{n}\right)-x\left(t_{m}+w_{k}\right)\right\| \leq a_{n, m}+a_{k, m}
$$

where $a_{n, m}=M \int_{-\infty}^{t_{m}} e^{-\omega\left(t_{m}-\tau\right)}\left\|f\left(\tau+w_{n}\right)-g(\tau)\right\| d \tau, \forall n, m \in \mathbb{N}$.

The uniform boundedness of the sequence of linear maps,

$$
\Theta_{m}(h):=\int_{-\infty}^{t_{m}} e^{-\omega\left(t_{m}-\tau\right)} h(\tau) d \tau, \forall h \in B C(\mathbb{R})
$$

and the fact that lemma (3.6) applies to $f_{w_{n}}-g\left(\right.$.), leads to $\left\|f_{w_{n}}-g().\right\| \rightarrow B C(\mathbb{R})$.

By going over to appropriate subsequences, we can assume that the iterated double limits for $\left(a_{n, m}\right)_{n, m \in \mathbb{N}}$ exist. Since they coincide, they have to be zero. By the triangle inequality we find,

$$
\left|\left\langle x\left(t_{m}+w_{n}\right)-x\left(t_{m}+w_{k}\right), x_{m}^{\star}\right\rangle\right| \leq a_{n, m}+a_{k, m} .
$$

Starting with $\lim _{k \rightarrow+\infty}, \lim _{m \rightarrow+\infty}$, at last $\lim _{n \rightarrow+\infty}$ we obtain

$$
\alpha=\beta,
$$

which proof that $x(.) \in W(\mathbb{R}, X)$.

it remains to prove the compactness of

$$
\{x(t): t \in \mathbb{R}\} .
$$

Thus for a given sequence $\left(t_{n}\right)_{n \in \mathbb{N}}$, we have to find a subsequence $\left(t_{n_{k}}\right)_{k \in \mathbb{N}}$ such that $\left(x\left(t_{n_{k}}\right)_{k \in \mathbb{N}}\right.$ is convergent in $X$. Since $f\left(\right.$.) has compact range, as a consequence of lemma (3.6), we may pass to subsequence $\left(t_{n_{k}}\right)_{k \in \mathbb{N}}$ of $\left(t_{n}\right)_{n \in \mathbb{N}}$, such that the limit of $\left(f_{t_{n_{k}}}(.)\right)_{k \in \mathbb{N}}$ exists in the weak topology of $B C(\mathbb{R}, X)$, and without loss of generality, we assume that

$$
f_{t_{n}} \rightarrow g
$$


with $g \in W R C(\mathbb{R}, X)$ and

$$
\left\|f_{t_{n}}-g(.)\right\| \rightarrow B C\left(\mathbb{R}^{+}\right)
$$

hence

$$
\begin{aligned}
\left\|x\left(t_{n}\right)-\int_{0}^{\infty} U(s) g(-s) d s\right\| & \leq \int_{0}^{\infty}\left\|U(s)\left(f\left(t_{n}-s\right)-g(-s)\right)\right\| d s \\
& \leq M \int_{0}^{\infty} e^{-\omega s}\left\|f\left(t_{n}-s\right)-g(-s)\right\| d s
\end{aligned}
$$

Thus,

$$
\left\|x\left(t_{n}\right)-\int_{0}^{\infty} U(s) g(-s) d s\right\| \leq c_{n}
$$

where $\forall n \in \mathbb{N}, \quad c_{n}:=\int_{0}^{\infty} e^{-\omega \tau}\left\|f\left(t_{n}-s\right)-g(-s)\right\| d s$. Consequently, by using remark (5), we deduce the desired result.

In the next, we study the existence and uniqueness of solutions in $W(\mathbb{R}, X)$ for equation (1).

Theorem 3.7. Assume that $\left(H_{1}\right)$ and $\left(H_{2}\right)$ hold. Let $f \in W R C(\mathbb{R} \times X, X)$ satisfy the following Lipschitz condition

$$
\|f(t, x)-f(t, y)\| \leq k\|x-y,\| \text { for } t \in \mathbb{R} \text { and } x, y \in X .
$$

Then, if $k<\frac{\omega}{M}$, the equation (1) has an unique mild solution $x(.) \in W R C(\mathbb{R}, X)$.

Proof. Let $K: W R C(\mathbb{R}, X) \rightarrow W R C(\mathbb{R}, X)$ by

$$
K(x(.))(t):=\int_{-\infty}^{t} U(t-r) f(r, x(r)) d r, \forall t \in \mathbb{R} .
$$

For $x_{1}(),. x_{2}(.) \in W R C(\mathbb{R}, X)$ and $t \in \mathbb{R}$ we have

$$
\begin{aligned}
\left\|K\left(x_{1}(.)\right)(t)-K\left(x_{2}(.)\right)(t)\right\| & \leq \int_{-\infty}^{t}\left\|U(t-r)\left[f\left(r, x_{1}(r)\right)-f\left(r, x_{2}(r)\right)\right]\right\| d r, \\
& \leq k M \int_{-\infty}^{t} e^{-\omega(t-r)}\left\|x_{1}(r)-x_{2}(r)\right\| d r, \\
& =k M\left\|x_{1}(.)-x_{2}(.)\right\|_{\infty} \int_{0}^{+\infty} e^{-\omega r} d r, \\
& =\frac{k M}{\omega}\left\|x_{1}(.)-x_{2}(.)\right\|_{\infty} .
\end{aligned}
$$

Using the contraction principle, we get that $K$ has a unique fixed point $x($.$) in W R C(\mathbb{R}, X)$.

\section{Example}

For illustration, we propose to study the following partial differential equation involving infinite delay:

$$
\left\{\begin{array}{l}
\frac{\partial u}{\partial t}(t, x)=\frac{\partial^{2} u}{\partial x^{2}}(t, x)+\int_{-\infty}^{t} e^{-(t-s)} \frac{\partial^{2} u}{\partial x^{2}}(s, x) d s+f(t, u(t)) \text { for } x \in[0, \pi], t \in \mathbb{R} \\
u(0, t)=u(\pi, t)=0 \text { for } t \in \mathbb{R} .
\end{array}\right.
$$


Let $X=L^{2}[0, \pi]$ and define $A:=\frac{\partial^{2} u}{\partial x^{2}}$, with domain $D(A)=\left\{g \in H^{2}[0, \pi]: g(0)=g(\pi)=0\right\}$. Then (6) can be written into the abstract form (1) with $\alpha=\beta=1$. It is well known that $A$ generates an immediately norm continuous $C_{0}$-semigroup $(T(t))_{t \geq 0}$ on $X=L^{2}[0, \pi]$ and $\sigma(A)=\left\{-n^{2}: n \in \mathbb{N}\right\}$. Since we must have $\lambda(\lambda+\beta)(\lambda+\alpha+\beta)^{-1} \in \sigma(A)$. We have to solve the equations $\frac{\lambda(\lambda+1)}{\lambda+2}=-n^{2}$, obtaining (see [7])

$$
\lambda_{1}=-1 \pm i, \quad \lambda_{2}=\frac{-5 \pm 7 i}{2}
$$

and

We conclude that

$$
\lambda_{n}=\frac{-\left(n^{2}+1\right) \pm \sqrt{\left(n^{2}-3\right)^{2}-8}}{2} \leq-2, \forall n \geq 3
$$

$$
\sup \left\{\operatorname{Re}(\lambda), \lambda \in \mathbb{C}: \lambda(\lambda+\beta)(\lambda+\alpha+\beta)^{-1} \in \sigma(A)\right\}=-1<0 .
$$

Hence, by theorem (3.2), there exist $M, \omega>0$ such that $\|U(t)\| \leq M e^{-\omega t}, \forall t \geq 0$ and from Theorem we obtain that if $f \in W R C(\mathbb{R} \times X, X)$ satisfies

$$
\|f(t, x)-f(t, y)\| \leq k\|x-y,\| \text { for } t \in \mathbb{R} \text {, and } x, y \in X
$$

where $k<\frac{\omega}{M}$, then equation (6) has a unique mild solution in $W R C(\mathbb{R}, X)$. In Particular case, if $f(t, \rho)(s)=$ $b(t) \sin \rho(s)$,for all $\rho \in X, t \in \mathbb{R}$ with $b(.) \in W R C(\mathbb{R}, X)$, then $t \rightarrow f(t, \rho)$ belongs to $W R C(\mathbb{R}, X)$, for each $\rho \in X$, and we have that

$$
\left\|f\left(t, \rho_{1}\right)-f\left(t, \rho_{2}\right)\right\|_{2}^{2} \leq \int_{0}^{\pi}|b(t)|^{2}\left|\sin \left(\rho_{1}(s)\right)-\sin \left(\rho_{2}(s)\right)\right|^{2} d s \leq\|b\|_{\infty}^{2}\left\|\rho_{1}-\rho_{2}\right\|_{2}^{2} .
$$

Then, we deduce that equation (6) has a unique mild solution in $W R C(\mathbb{R}, X)$ provided that $\|b\|_{\infty}$ is small enough.

Acknowledgement: The authors were partially supported by the IRD project UMMISCO

The authors would like to thank the referees for their careful reading of the paper. Their valuable suggestions made numerous improvements throughout.

\section{References}

[1] E. Ait Dads E, K. Ezzinbi and S. Fatajou, Weakly almost periodic solutions for some differential equations in a Banach space, Nonlinear Studies, 42 (1997) 157 - 170.

[2] E. Ait Dads E, K. Ezzinbi and S. Fatajou, Weakly almost periodic solutions for the inhomogeneous linear equations and periodic processes in a Banach space, Dynamic Systems and Applications, 6 (1997), 507 - 516.

[3] E. Ait Dads E, K. Ezzinbi and S. Fatajou, Asymptotic behaviour of solutions for some differential equations in Banach spaces, Afr. Diapora J. Math., 12 1(2011), 1 - 18.

[4] J. F. Berglund, H. D. Junghenn and P. Milnes, Analysis on Semigroups, Wiley Inerscience, 1989.

[5] S. Bochner, A new approach to almost periodicity, Proc. Nat. Acad. Sci. USA 48 (1962), 2039 - 2043.

[6] H. Bohr, Zur theorie der fastperiodisch functionen : Eine Versall gemeinerung det theorie der Fourierreihen. Acta. Math. Bd. 45 (1925), $29-127$.

[7] J. Chen, T. Xiao, and J. Liang, Uniform exponential stability of solutions to abstract Volterra equations, J. Evol. Equ. 4 9 (2009), $661-674$.

[8] W. F. Eberlein, Abstract ergodic theorems and weak almost periodic functions, TAMS. 67 (1949), $217-240$.

[9] T. Diagana, G. M. Mophou and G. M. N'Guérékata, On the existence of mild solutions to some semilinear fractional integrodifferential equations, Electron. J. Qual. Theory Differ. Equ. 58 (2010), 1 - 17.

[10] T. Diagana and G.M. N'Guérékata, Almost automorphic solutions to some classes of partial evolution equations, 86 6 (2007), $462-466$.

[11] T. Diagana, Stepanov-like pseudo-almost periodicity and its applications to some nonautonomous differential equations, Nonlinear Analysis 69(2008), $4277-4285$. 
[12] T. Diagana, H. R. Henriquez and E. M. Hernandez, Almost automorphic mild solutions to some partial neutral functionaldifferential equations and applications, Nonlinear Analysis : Theory, Methods and Applications, 695 (2008), 1485-1493.

[13] T. Diagana, Pseudo almost periodic functions in Banach spaces, Nova Science Publishers, Inc., New York, 2007.

[14] K. De Leeuw and I. Glicksberg, Applications of almost periodic compactifications, Acta Math, 105 (1961), 63 - 97.

[15] K. De Leeuw and I. Glicksberg, Almost periodic functions on semigroups, Acta Math, 105 (1961), 99 - 140.

[16] N. Dunford and J. T. Schwartz, Linear Operators, John Wiley and Sons, Vol 1. (1959).

[17] C. F. Dunkl and D. E. Ramirez, Topics in Harmonic Analysis, Appleton-Centry-Crofts, New York, 1971.

[18] A. Grothendiek, Critères de compacité dans les espaces fonctionels généraux, Amer. J. Math. 74 (1952), 168 - 186.

[19] U. Krengel, Ergodic Theorems, De Gruyter Studies in Math. 1985.

[20] C. Lizama and V. Poblete, Maximal regularity for perturbed integral equations on periodic Lebesgue spaces, J. Math. Anal. 3482 (2008), $775-786$.

[21] C. Lizama and R. Ponce, Bounded solutions to a class of semilinear integro-differential equations in Banach spaces, Nonlinear Analysis., 74 (2011), 3397 - 3406.

[22] C. Lizama and R. Ponce, Almost automorphic solutions to abstract Volterra equations on the line, Nonlinear Analysis., 74 (2011), $3805-3814$.

[23] M. A. Meyers and K. K. Chawla, Mechanical Behavior of Materials (Second Edition), Cambridge University, Wiley, New York (2009).

[24] G. M. N'Guérékata, Existence and uniqueness of almost automorphic mild solutions of some semilinear abstract differential equations, Semigroup Forum, 69 (2004), 80 - 86.

[25] G. M. N'Guérékata, Topics in Almost Automorphy, Springer Verlag, New York, (2005).

[26] G. M. N'Guérékata, Almost automorphic solutions to second-order semilinear evolution equations, Nonlinear Anal., 71 (2009), e432-e435.

[27] V. Poblete, Solutions of second-order integro-differential equations on periodic Besov spaces, Proc. Edinb. Math. Soc., 50 2 (2007), $477-492$.

[28] G. Da Prato, A. Lunardi, Periodic solutions for linear integro-differential with infinite delay in Banach space, Differential Equations in Banach spaces, Lecture notes in Math. 1223 (1985), 49 - 60.

[29] J. Pruss, Evolutionary Integral Equations and Applications, Monographs Math., 87 Birkhauser Verlag, (1993).

[30] W.Rudin, Weak almost periodic functions and Fourier Stieljes transforms, Duke Math. J. 26 (1959), 215 - 220.

[31] W. M. Ruess, W. H. Summers, Integration of asymptotically almost periodic functions and weak asymptotic almost periodicity, Dissertationes Math., 279 (1989).

[32] W. M. Ruess and W. H. Summers, Weak almost periodicity and the strongly ergodiclLimit Theorem for periodic evolution systems, J.Funct. Anal., 94 (1990), $177-195$.

[33] W. M. Ruess and W. H. Summers, Weak almost periodic semigroups of operators, Pacific J. Math., 43 (1990), 175 - 193.

[34] W. M. Ruess and W. H. Summers, Ergodic theorems for semigroups of operators, Proc. Amer. Math. Soc. 114 (1992), $423-$ 432. 